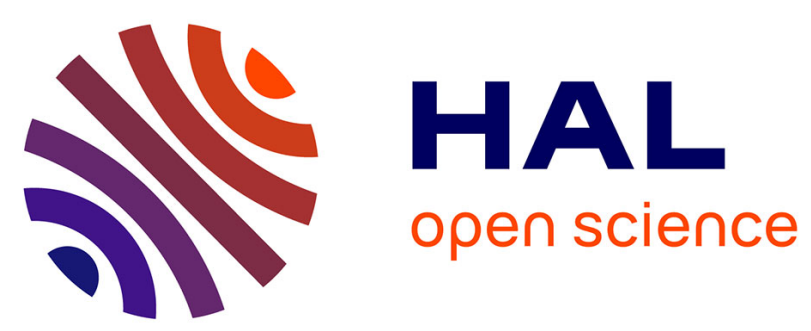

\title{
Théorème de Jordan friable
}

Régis de La Bretèche, Gérald Tenenbaum

\section{To cite this version:}

Régis de La Bretèche, Gérald Tenenbaum. Théorème de Jordan friable. C. Pomerance, M. Rassias. Analytic number theory, volume in honor of Helmut Maier, Springer, pp.57-64, 2015. hal-01281592

\section{HAL Id: hal-01281592 \\ https://hal.science/hal-01281592}

Submitted on 2 Mar 2016

HAL is a multi-disciplinary open access archive for the deposit and dissemination of scientific research documents, whether they are published or not. The documents may come from teaching and research institutions in France or abroad, or from public or private research centers.
L'archive ouverte pluridisciplinaire HAL, est destinée au dépôt et à la diffusion de documents scientifiques de niveau recherche, publiés ou non, émanant des établissements d'enseignement et de recherche français ou étrangers, des laboratoires publics ou privés. 


\title{
Théorème de Jordan friable
}

\author{
R. de la Bretèche \& G. Tenenbaum
}

Pour Helmut Maier, qui compte par plaisir et partage sans compter.

\begin{abstract}
Extending a previous result, we show that, for the friable summation method, the Fourier series of any normalized function $F$ with bounded variation on the unidimensional torus converges pointwise to $F$ while avoiding the Gibbs phenomenon. We also prove that the convergence is uniform when $F$ is continuous and provide an effective bound for the speed when $F$ satisfies a uniform Lipschitz condition.

Keywords: Friable integers, friable summation, summation methods, Fourier series, Gibbs phenomenon, functions of bounded variation, Jordan's theorem, integers free of large prime factors.
\end{abstract}

\section{Introduction et énoncé des résultats}

Soit $V B^{*}(\mathbb{T})$ la classe des fonctions $F$ qui sont 1-périodiques, à variation bornée $\operatorname{sur} \mathbb{T}=\mathbb{R} / \mathbb{Z}$, et normalisées par $F(\vartheta)=\frac{1}{2}\{F(\vartheta+)+F(\vartheta-)\}$ aux points de discontinuité. Nous nous proposons ici de généraliser aux fonctions de $V B^{*}(\mathbb{T})$ le théorème 5.1 de [2] relatif à la convergence friable des séries de Fourier et à l'absence de phénomène de Gibbs (voir par exemple, [9], vol. I, §II.9, ou [6], chap. 17) pour ce procédé de sommation.

Nous notons $a_{n}(F), b_{n}(F)$ les coefficients de Fourier d'une fonction $F$ de $L^{1}(\mathbb{T})$, et nous définissons la suite des sommes partielles friables de la série de Fourier de $F$ par

$$
F(\vartheta ; y):=a_{0}(F)+\sum_{P(n) \leqslant y}\left\{a_{n}(F) \cos (2 \pi n \vartheta)+b_{n}(F) \sin (2 \pi n \vartheta)\right\} \quad(y \geqslant 2)
$$

pour toute valeur de $\vartheta$ où cela possède un sens. C'est en particulier le cas si $F \in V B^{*}(\mathbb{T})$ puisque $\left|a_{n}(F)\right|+\left|b_{n}(F)\right| \ll 1 / n$, ce qui implique l'absolue convergence de la série $(1 \cdot 1)$ pour chaque $y \geqslant 2$.

L'étude de la convergence de $F(\vartheta ; y)$ lorsque $y$ tend vers l'infini s'inscrit dans le cadre plus général de celle de la convergence friable des séries. Formellement introduit dans [3] puis dans [4], ce procédé de sommation ${ }^{(1)}$ consistant à définir la somme comme limite, lorsque le paramètre de friabilité tend vers l'infini, des sous-séries restreintes aux entiers friables, a été étudié dans [1] puis de manière systématique dans [2]. Au théorème 5.1 de [2], nous avons établi la convergence simple de $F(\vartheta ; y)$ vers $F(\vartheta)$ pour toute fonction $F$ de $V B^{*}(\mathbb{T})$ sous réserve que la dérivée presque partout de $F$ soit dans $\cup_{\alpha>1} L^{\alpha}(\mathbb{T})$. Le but de la présente note consiste à relâcher cette dernière condition.

Il est relativement facile d'exhiber des exemples de fonctions discontinues $F$ dans $V B^{*}(\mathbb{T}) \backslash \cup_{\alpha>1} L^{\alpha}(\mathbb{T}):$ si $f \in L^{1}(\mathbb{T}) \backslash \cup_{\alpha>1} L^{\alpha}(\mathbb{T})$, on peut choisir

$$
F(\vartheta):=\int_{0}^{\vartheta} f(t) \mathrm{d} t+B(\vartheta)
$$

Un exemple de fonction $f$ admissible est fourni par

$$
f(\vartheta):=\sum_{n \geqslant 2} \frac{\cos (2 \pi n \vartheta)}{\log n} .
$$

En effet, un théorème classique concernant les séries trigonométriques $\sum_{n \in \mathbb{Z}} a_{n} \mathrm{e}^{2 \pi i n \vartheta}$ dont la suite des coefficients est paire et vérifie identiquement $a_{n-1}+a_{n+1} \geqslant 2 a_{n}$ (cf. [5],

2010 Mathematics Subject Classification: primary 11N25, 42A24; secondary 42A20.

1. Désigné dans ces travaux sous le nom de $P$-convergence ou $P$-sommabilité. 
th. I.4.1, ou [9], vol. I, th. V.1.5) implique $f \in L^{1}(\mathbb{T}),{ }^{(2)}$ que alors que, pour tout $\alpha>1$, posant $r:=\min (2, \alpha), s:=r /(r-1)$, l'hypothèse $f \in L^{\alpha}(\mathbb{T})$ impliquerait $f \in L^{r}(\mathbb{T})$ et donc (cf., par exemple, [5], th. I.4.7)

$$
\sum_{n \in \mathbb{Z}}|\widehat{f}(n)|^{s}<\infty
$$

une condition manifestement en défaut pour la série $(1 \cdot 2)$.

Nous désignons par $\varrho$ la fonction de Dickman, ${ }^{(3)}$ posons $\|v\|:=\min _{n \in \mathbb{Z}}|v-n|(v \in \mathbb{R})$ et notons

$$
B(\vartheta ; y):=-\sum_{P(n) \leqslant y} \frac{\sin (2 \pi n \vartheta)}{\pi n}
$$

la somme partielle friable d'ordre $y$ de la série de Fourier de la fonction de Bernoulli

$$
B(\vartheta):=-\sum_{m \geqslant 1} \frac{\sin (2 \pi m \vartheta)}{\pi m}= \begin{cases}\langle\vartheta\rangle-\frac{1}{2} & \text { si } \vartheta \notin \mathbb{Z} \\ 0 & \text { si } \vartheta \in \mathbb{Z} .\end{cases}
$$

où $\langle\vartheta\rangle$ désigne la partie fractionnaire du nombre réel $\vartheta$.

Théorème 1.1. Soit $F \in V B^{*}(\mathbb{T})$. On a, uniformément pour $\vartheta \in \mathbb{R}$ et $y \geqslant 2$,

$$
F(\vartheta ; y)-F(\vartheta)=\int_{\mathbb{T}}\left\{\varrho\left(\frac{\log (1 /\|v\|)}{\log y}\right)-1\right\} B(v) \mathrm{d} F(\vartheta-v)+O\left(\frac{1}{\log y}\right) .
$$

En particulier, on a

$$
\begin{aligned}
\lim _{y \rightarrow \infty} F(\vartheta ; y) & =F(\vartheta) \quad(\vartheta \in \mathbb{T}), \\
\lim _{y \rightarrow \infty} \sup _{\vartheta \in \mathbb{T}} F(\vartheta ; y) & =\sup _{\vartheta \in \mathbb{T}} F(\vartheta) .
\end{aligned}
$$

De plus, si $F$ est continue, alors $F(\cdot ; y)$ tend uniformément vers $F$.

Remarque. Bien que l'on ait $(1 \cdot 7)$, la convergence de $F(\vartheta ; y)$ vers $F(\vartheta)$ n'est en général pas uniforme : il est facile de voir que la série $F(\vartheta ; y)$ converge uniformément et donc que, pour chaque $y \geqslant 2$ fixé, $F(\vartheta ; y)$ dépend continûment de $\vartheta$. Il est cependant à noter que, contrairement à la situation classique, la convergence demeure exploitable au voisinage des discontinuités : alors que l'on sait, classiquement, que la somme partielle d'ordre $y$ de la série $(1 \cdot 4)$ n'approche pas le membre de gauche pour, par exemple, $\vartheta=1 / y$, on déduit de $(1.5)$ que

$$
B(1 / y ; y)-B(1 / y) \ll 1 / \log y \quad(y \geqslant 2) .
$$

Sous une hypothèse supplémentaire de régularité pour $F$, nous pouvons préciser la vitesse de convergence de $F(\cdot ; y)$ vers $F$.

Théorème 1.2. Soit $\alpha>0$. Si $F \in V B^{*}(\mathbb{T})$ est uniformément lipschitzienne d'exposant $\alpha>0$, on a

$$
\max _{\vartheta \in \mathbb{T}}|F(\vartheta ; y)-F(\vartheta)| \ll \frac{1}{y^{\alpha / 2}} \quad(y \geqslant 2) .
$$

2. On peut aussi établir directement via la formule d'Euler-Maclaurin que $f(\vartheta) \ll 1 /\left\{\vartheta(\log \vartheta)^{2}\right\}$ pour $0<|\vartheta| \leqslant \frac{1}{2}$.

3. Voir par exemple [7], chap. III.5. 


\section{Preuve du Théorème $\mathbf{1 . 1}$}

Pour toute fonction $F$ de $V B^{*}(\mathbb{T})$ et tout entier $n \geqslant 1$, on a

$$
\begin{aligned}
a_{n}(F) \cos (2 \pi n \vartheta) & +b_{n}(F) \sin (2 \pi n \vartheta)=2 \int_{\mathbb{T}} \cos (2 \pi n(\vartheta-v)) F(v) \mathrm{d} v \\
& =-\int_{\mathbb{T}} \frac{\sin (2 \pi n(\vartheta-v))}{\pi n} \mathrm{~d} F(v)=-\int_{\mathbb{T}} \frac{\sin (2 \pi n v)}{\pi n} \mathrm{~d} F(\vartheta-v) .
\end{aligned}
$$

Cette quantité étant trivialement $\ll 1 / n$, on peut sommer pour $P(n) \leqslant y$. On en déduit que l'on a pour tout $y \geqslant 2$

$$
F(\vartheta ; y)=a_{0}(F)+\int_{\mathbb{T}} B(v ; y) \mathrm{d} F(\vartheta-v) .
$$

Par ailleurs, une simple intégration par parties fournit, dès que $F \in V B^{*}(\mathbb{T})$,

$$
F(\vartheta)=a_{0}(F)+\int_{\mathbb{T}} B(v) \mathrm{d} F(\vartheta-v) .
$$

En effectuant la différence de $(2 \cdot 1)$ et $(2 \cdot 2)$, nous obtenons

$$
F(\vartheta ; y)-F(\vartheta)=\int_{\mathbb{T}} \nabla_{\mathbf{1}}(v ; y) \mathrm{d} F(\vartheta-v),
$$

où l'on a posé

$$
\nabla_{\mathbf{1}}(\vartheta ; y):=B(\vartheta ; y)-B(\vartheta)
$$

Or, nous avons établi dans [2] que l'on a, uniformément pour $\vartheta \in \mathbb{R}$,

$$
\nabla_{\mathbf{1}}(\vartheta ; y)=\left\{\varrho\left(u_{\vartheta, y}^{*}\right)-1\right\} B(\vartheta)+O\left(\frac{1}{\log y}\right),
$$

où nous avons posé

$$
u_{\vartheta, y}^{*}:=\frac{\log (1 /\|\vartheta\|)}{\log y} \quad(\vartheta \in \mathbb{R} \backslash \mathbb{Z})
$$

et convenu que $u_{\vartheta, y}^{*}=\infty$ si $\vartheta \in \mathbb{Z}$. Il s'ensuit que

$$
F(\vartheta ; y)-F(\vartheta)=\int_{\mathbb{T}}\left\{\varrho\left(u_{v, y}^{*}\right)-1\right\} B(v) \mathrm{d} F(\vartheta-v)+O_{F}(1 / \log y) .
$$

Comme $\left\{\varrho\left(u_{v, y}^{*}\right)-1\right\} B(v)$ tend simplement vers 0 sur $\mathbb{T}$, la relation (1.5) implique clairement (1.6), en vertu du théorème de la convergence dominée.

Montrons maintenant (1.7). Il résulte immédiatement de (1.6) que

$$
\limsup _{y \rightarrow \infty} \sup _{\vartheta} F(\vartheta ; y) \geqslant \sup _{\vartheta} F(\vartheta) \text {. }
$$

Il suffit donc de prouver l'inégalité inverse. Nous pouvons supposer sans perte de généralité que $a_{0}(F)=0$. Posons

$$
F_{y}(\vartheta):=\int_{\mathbb{T}} \varrho\left(u_{v, y}^{*}\right) B(v) \mathrm{d} F(\vartheta-v),
$$

de sorte que, par $(2 \cdot 2)$ et $(2 \cdot 6)$, on a

$$
\sup _{\vartheta}\left|F(\vartheta ; y)-F_{y}(\vartheta)\right| \ll 1 / \log y \quad(y \rightarrow \infty) .
$$

Comme l'application $v \mapsto \varrho\left(u_{v, y}^{*}\right) B(v)$ est continue sur $\mathbb{T}, F_{y}(\vartheta)$ est, pour chaque $y$, une fonction continue de $\vartheta$. Elle atteint donc son maximum en un point $\vartheta_{y}$. Quitte à extraire une sous-suite, nous pouvons supposer que

$$
\limsup _{y \rightarrow \infty} \sup _{\vartheta} F(\vartheta ; y)=\lim _{y \rightarrow \infty} F_{y}\left(\vartheta_{y}\right)
$$

et que $\vartheta_{y}$ tend vers une limite $\vartheta_{0} \in \mathbb{T}$. Quitte à changer $F(\vartheta)$ en $F\left(\vartheta-\vartheta_{0}\right)$, nous pouvons encore effectuer l'hypothèse que $\vartheta_{0}=0$. 
Soit $\delta_{F}:=F(0+)-F(0-)$ le saut de $F$ en 0 . Posons

$$
H(\vartheta):=1+\lfloor\vartheta\rfloor-\frac{1}{2} \mathbf{1}_{\mathbb{Z}}(\vartheta)
$$

de sorte que $\widetilde{F}:=F-\delta_{F} H$ est continue sur $\mathbb{Z}$. Pour $\vartheta \in \mathbb{T}$, nous avons

$$
F_{y}(\vartheta)=\int_{\mathbb{T}} \varrho\left(u_{v, y}^{*}\right) B(v) \mathrm{d} \widetilde{F}(\vartheta-v)+\delta_{F} \varrho\left(u_{\vartheta, y}^{*}\right) B(\vartheta) .
$$

Comme $\varrho\left(u_{v, y}^{*}\right) B(v)$ tend simplement vers $B(v)$ sur $\mathbb{T}$ avec une convergence uniforme sur tout compact de $\mathbb{T}$ ne contenant pas 0 , et comme $\widetilde{F}$ est continue en 0 , on a

$$
\lim _{y \rightarrow \infty} \int_{\mathbb{T}} \varrho\left(u_{v, y}^{*}\right) B(v) \mathrm{d} \widetilde{F}\left(\vartheta_{y}-v\right)=\int_{\mathbb{T}} B(v) \mathrm{d} \widetilde{F}(-v) .
$$

Il suit

$$
F_{y}\left(\vartheta_{y}\right) \leqslant \int_{\mathbb{T}} B(v) \mathrm{d} \widetilde{F}(-v)+\frac{1}{2}\left|\delta_{F}\right|+o(1) .
$$

Or, en faisant tendre $y$ vers l'infini dans $(2 \cdot 7)$, nous obtenons successivement

$$
F(0 \pm)=\int_{\mathbb{T}} B(v) \mathrm{d} \widetilde{F}(-v) \mp \frac{1}{2} \delta_{F}, \quad \max \{F(0+), F(0-)\}=\int_{\mathbb{T}} B(v) \mathrm{d} \widetilde{F}(-v)+\frac{1}{2}\left|\delta_{F}\right| .
$$

On en déduit que

$$
F_{y}\left(\vartheta_{y}\right) \leqslant \max \{F(0+), F(0-)\}+o(1) \leqslant \sup _{\vartheta} F(\vartheta)+o(1) .
$$

Cela achève la preuve de $(1 \cdot 7)$.

Lorsque $F$ est continue, et puisque $\varrho(v)=1$ pour $0 \leqslant v \leqslant 1$, la relation (1.5) implique

$$
|F(\vartheta ; y)-F(\vartheta)| \leqslant \int_{\vartheta-1 / y}^{\vartheta+1 / y}|\mathrm{~d} F|(t)+O\left(\frac{1}{\log y}\right),
$$

où la constante implicite ne dépend que de $F$. Comme $F$ est continue, et donc uniformément continue en vertu de la compacité du tore, il en va de même de sa variation. La dernière intégrale tend donc vers 0 uniformément en $\vartheta$.

\section{Preuve du Théorème $\mathbf{1 . 2}$}

Sous l'hypothèse que $F$ est à variation bornée sur $\mathbb{T}$ et satisfait une condition de Lipschitz uniforme

$$
F(\vartheta+h)-F(\vartheta) \ll|h|^{\alpha},
$$

où $\alpha>0$ et la constante implicite est indépendante de $\vartheta \in \mathbb{T}$, un théorème de Zygmund ([9], vol. I, th. VI.3.6) implique que la série de Fourier de $F$, i.e.

$$
F(\vartheta)=\sum_{n \in \mathbb{Z}} c_{n}(F) \mathrm{e}^{2 \pi i n \vartheta}
$$

est absolument convergente.

En fait, la démonstration de Zygmund fournit, sous la seule hypothèse que $F$ est à variation bornée, la majoration

$$
\sum_{2^{\nu-1}<|n| \leqslant 2^{\nu}}\left|c_{n}(F)\right| \leqslant \frac{1}{2} \sqrt{V_{F} \omega_{F}\left(1 / 2^{\nu+1}\right)} \quad\left(\nu \in \mathbb{N}^{*}\right),
$$

où $V_{F}$ désigne la variation totale de $F$ sur $\mathbb{T}$ et $h \mapsto \omega_{F}(h)$ son module de continuité. 
Pour la commodité du lecteur, nous reproduisons ici la courte preuve de [9]. Soient $\nu \geqslant 1, N:=2^{\nu+1}$. Pour tout $\vartheta \in \mathbb{T}$, nous avons

$$
\sum_{1 \leqslant k \leqslant N}\left|F\left(\vartheta+\frac{k}{N}\right)-F\left(\vartheta+\frac{k-1}{N}\right)\right|^{2} \leqslant V_{F} \omega_{F}\left(\frac{1}{N}\right) .
$$

En intégrant sur $\mathbb{T}$, nous obtenons

$$
4 N \sum_{n \in \mathbb{Z}}\left|c_{n}(F)\right|^{2} \sin ^{2}\left(\frac{\pi n}{N}\right) \leqslant V_{F} \omega_{F}\left(\frac{1}{N}\right)
$$

Nous en déduisons (3·3) en observant que, pour $N / 4<|n| \leqslant N / 2$, nous avons

$$
\sqrt{2} / 2 \leqslant|\sin (\pi n / N)| \leqslant 1
$$

et en appliquant l'inégalité de Cauchy-Schwarz.

Sous la condition $(3 \cdot 1)$, nous déduisons immédiatement de $(3 \cdot 3)$ que

$$
\sum_{|n|>y}\left|c_{n}(F)\right| \ll_{F} \frac{1}{y^{\alpha / 2}} \quad(y \geqslant 1) .
$$

Posons

$$
F_{N}(\vartheta):=\sum_{|n| \leqslant N} c_{n}(F) \mathrm{e}(n \vartheta), \quad F_{N}(\vartheta ; y):=\sum_{\substack{|n| \leqslant N \\ P(|n|) \leqslant y}} c_{n}(F) \mathrm{e}(n \vartheta) \quad(N \geqslant 1, \vartheta \in \mathbb{T}, y \geqslant 2)
$$

Pour tous $\vartheta \in \mathbb{T}, N \geqslant 1$, nous avons, par $(2 \cdot 3)$,

$$
F_{N}(\vartheta ; y)-F_{N}(\vartheta)=-\sum_{\substack{|n| \leqslant N \\ P(|n|)>y}} c_{n}(F) \mathrm{e}(n \vartheta) \ll \frac{1}{y^{\alpha / 2}},
$$

où la majoration découle de $(3 \cdot 4)$, la constante implicite étant donc indépendante de $N$.

Comme $F \in V B^{*}(\mathbb{T})$, on a $c_{n}(F) \ll 1 / n$ pour tout $n \in \mathbb{Z}^{*}$, donc $F_{N}(\vartheta ; y)$ tend simplement vers $F(\vartheta ; y)$ lorsque $N$ tend vers l'infini. D'après le théorème de Jordan, il en va de même de la convergence de $F_{N}(\vartheta)$ vers $F(\vartheta)$. Nous obtenons donc (1.8) en faisant tendre $N$ vers l'infini dans (3.5).

\section{Bibliographie}

[1] R. de la Bretèche, P-régularité de sommes d'exponentielles, Mathematika, 45 (1998), 145-175.

[2] R. de la Bretèche \& G. Tenenbaum, Séries trigonométriques à coefficients arithmétiques, J. Anal. Math. 92 (2004), 1-79.

[3] R.J. Duffin, Representation of Fourier integrals as sums, III. Proc. Amer. Math. Soc. 8 (1957), $272-277$.

[4] E. Fouvry \& G. Tenenbaum, Entiers sans grand facteur premier en progressions arithmétiques, Proc. London Math. Soc. (3) 63 (1991), 449-494.

[5] Y. Katznelson, An introduction to harmonic analysis, Dover, New York, $2^{\mathrm{e}}$ édition, 1976 ( $1^{\mathrm{e}}$ éd. 1968).

[6] T.W. Körner, Fourier analysis, Second edition. Cambridge University Press, Cambridge, 1989. xii+591 pp. 
[7] G. Tenenbaum, Introduction à la théorie analytique et probabiliste des nombres, troisième édition, coll. Échelles, Belin, 2008, 592 pp.

[8] G. Tenenbaum \& J. Wu, Exercices corrigés de théorie analytique et probabiliste des nombres, coll. Échelles, Belin, 2014, à paraître.

[9] A. Zygmund, Trigonometric series, Vol. I, II, 3 ème édition, Cambridge Mathematical Library, Cambridge University Press, Cambridge, 2002 ; vol. I : xiv+383 pp. ; vol. II : viii+364 pp.

Régis de la Bretèche

Institut de Mathématiques de Jussieu

UMR 7586

Université Paris Diderot-Paris 7, case 7012

Bâtiment Sophie Germain

75205 Paris Cedex 13

France

regis.de-la-breteche@imj-prg.fr
Gérald Tenenbaum

Institut Élie Cartan

Université de Lorraine

BP 70239

54506 Vandœuvre-lès-Nancy Cedex

France

gerald.tenenbaum@univ-lorraine.fr 